\title{
Manufactura y pruebas de una celda de carga para un banco de pruebas de aspas de turbinas eólicas de baja capacidad
}

\section{Manufacturing and testing of a load cell for a bank of low capacity wind turbine test blocks}

ROMERO-FRANCO, Martín†*, LÓPEZ-GARZA, Víctor, MARÍN-TÉLLEZ, Paulina y PÉREZJUÁREZ, Erik

Universidad Michoacana de San Nicólas de Hidalgo

ID $1^{\mathrm{er}}$ Autor: Martín, Romero-Franco / ORC ID: 0000-0001-6494-9344, CVU CONACYT ID: 994229

ID $1^{\text {er }}$ Coautor: Víctor, López-Garza / ORC ID: 0000-0001-9090-9119, Researcher ID Thomson: H-6969-2018, CVU CONACYT ID: 554311

ID $2^{\text {do }}$ Coautor: Paulina, Marín-Téllez / ORC ID: 0000-0003-2966-3409, Researcher ID Thomson: G-2113-2019, CVU CONACYT ID: 730422

ID $3^{\text {er }}$ Coautor: Erik, Pérez-Juárez, / ORC ID: 0000-0003-3069-7198, Researcher ID Thomson: G-4108-2018, CVU CONACYT ID: 815741

\begin{abstract}
Resumen
En el presente trabajo, se muestra el diseño, manufactura y pruebas mecánicas de una celda de carga para aplicación en un banco de pruebas de aspas de turbinas eólicas de baja capacidad. El banco de pruebas, está diseñado para soportar cargas de hasta $3000 \mathrm{Nw}$ y álabes de hasta $2.5 \mathrm{~m}$ de longitud. La metodología general de diseño de la celda consiste en, primero determinar las cargas de operación del banco, la selección del tipo de celda de carga, la manufactura de ésta, la selección de las galgas extensométricas para la medición, la colocación de las galgas extensométricas en la celda de carga y finalmente las pruebas mecánicas de la celda en el banco de pruebas. El material de la celda es aluminio 6061 y la conFiguración es tipo "S". Como conclusiones se menciona que técnicas de extensométrica permiten caracterizar la celda de carga mediante pruebas en laboratorio cumpliendo con las especificaciones deseadas con el diseño.
\end{abstract}

Celda, Galga, Carga

\begin{abstract}
In the present work, it is shows the design, manufacture and mechanical tests of a load cell for application in a turbine test bench of low capacity wind turbines are shown. The test bench is designed to withstand loads up to $3000 \mathrm{Nw}$ and vanes up to $2.5 \mathrm{~m}$ in length. The general methodology of the cell design consists of, first, determining the operating loads of the bank, the selection of the type of load cell, the manufacture of this, the selection of the strain gauges for the measurement, the placement of the strain gauges in the load cell and finally the mechanical tests of the cell in the test bench. The material of the cell is aluminum 6061 and the conFiguration is type "S". As conclusions, it is mentioned that extensometric techniques allow to characterize the load cell by means of laboratory tests fulfilling the desired specifications with the design.
\end{abstract}

Citación: ROMERO-FRANCO, Martín, LÓPEZ-GARZA, Víctor, MARÍN-TÉLLEZ, Paulina y PÉREZ-JUÁREZ, Erik. Manufactura y pruebas de una celda de carga para un banco de pruebas de aspas de turbinas eólicas de baja capacidad. Revista de Ingeniería Mecánica. 2019. 3-11: 14-23

\footnotetext{
* Correspondencia al Autor (Correo electrónico: onizukadragon@ @otmail.com)

$\dagger$ Investigador contribuyendo como primer Autor.
} 


\section{Introducción}

Mediante el uso de galgas extensiométricas se pretende manufacturar una celda de carga tipo "S" para usarse en un banco de pruebas para aspas de turbinas eólicas de baja capacidad.

Haciendo uso de consideraciones de diseño se opto por la celda de carga de carga tipo "S" ya que nos permite obtener mediciones tanto a tensión como a compresión, para la cual se seleccionó la aleación de aluminio 6061.

Antes de su construcción se realizó un modelo en 3D, luego utilizando un software de elemento finito para obtener los datos de deformación y de esa manera hacer una comparación con los datos obtenidos mediante la medición de las galgas extensiométricas.

\section{Objetivos}

El objetivo principal es el diseño, simulación y manufactura de una celda de carga tipo "S" para poder medir fuerzas en aspas eólicas montadas en un banco de pruebas.

\section{Metodología}

Para crear el modelo en 3D se hará uso del software SOLIDWORDS para su construcción, después para realizar la simulación se usará un software de elemento finito con el cual se obtendrán datos de deformación bajo diferentes cargas simuladas, de forma silimar se realizará la manufactura de la celda de carga en aluminio 6061, posteriormente se le colocarán las galgas extensiométricas y conectara al equipo de medición P3, se le agregarán pesos, registrando los datos obtenidos.

\section{Contribución}

Este proyecto contribuye al área de investigación científica ya que se tomaron en cuenta las especificaciones de diseño y el modelo de la celda de carga para su uso en un banco de pruebas para aspas de baja capacidad, lo que significa que es una celda de carga personalizada para un uso en específico, la cual se puede ir adaptando según sea conveniente.

\section{Consideraciones de Diseño}

Para la creación del modelo en $3 \mathrm{D}$, primero se tuvo que hacer una investigación acerca de la celda de carga tipo "S", para lo que nos basaremos en la hoja de especificaciones para celdas de carga tipo "S" $D F Y-A /-A S S$ públicado por la empresa $K O L I$ y seleccionando las especificaciones correspondientes para pesos entre $2 \mathrm{klb}$ y $3 \mathrm{klb}$.

En la Figura 1 se muestra el diagrama con las especificaciones para una celda de carga tipo "S".
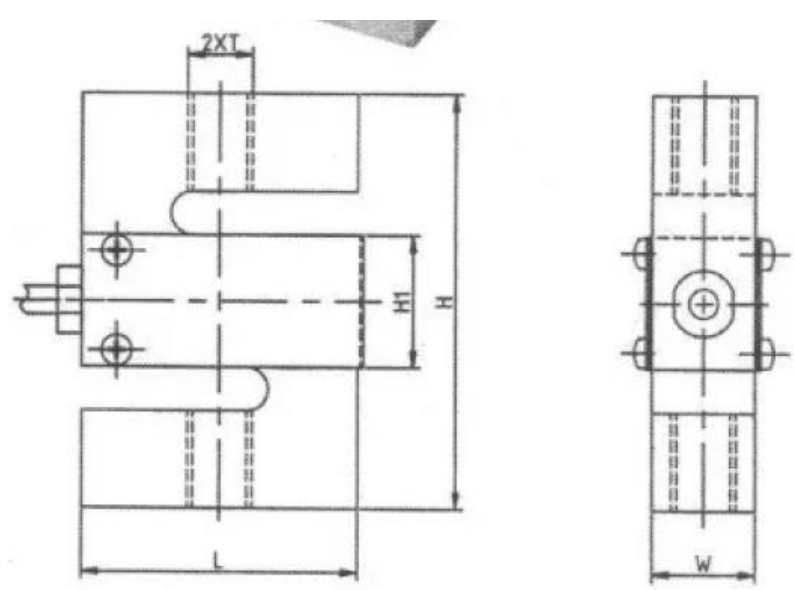

Figura 1 Dimensiones para una celda de carga tipo "S"

Siendo las dimensiones correspondientes:

L: $51 \mathrm{~mm}$

$\mathrm{W}: 19.1 \mathrm{~mm}$

$\mathrm{H}: 76.2 \mathrm{~mm}$

H1: $24 \mathrm{~mm}$

T: 1/2-24 UNF

Construcción del Modelo Digital en 3D, Mediante el Uso de Software de Diseño SOLIDWORKS

Ya teniendo las dimensiones necesarias se prosiguió a realizar al modelo en 3D de la celda de carga tipo "S", para lo que utilizó el software de diseño SOLIDWORKS, en la Figura 2 se muestra el modelo en $3 \mathrm{D}$ de la celda de carga tipo " $\mathrm{S}$ " terminado. 


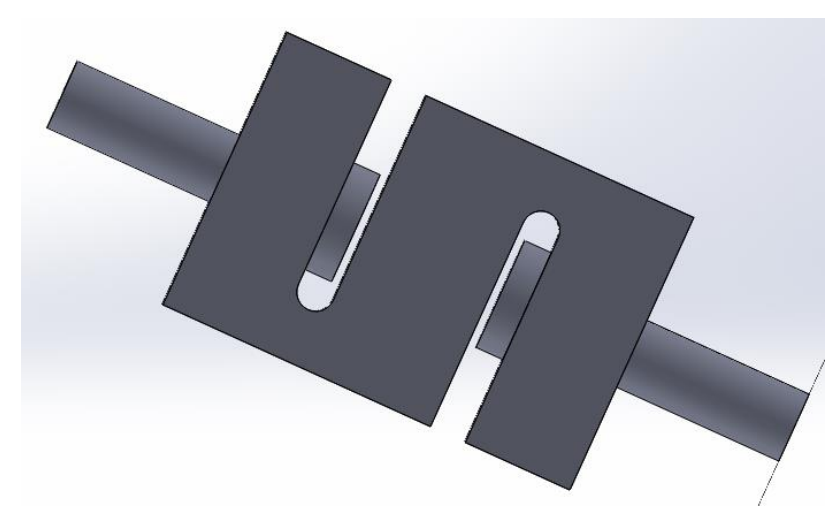

Figura 2 Modelo en 3D de la celda de carga construido en solidworks

Se debe señalar que no es necesario hacer las roscas de los barrenos y los tornillos ya que solo interesa la deformación en la superficie de la celda de carga, por lo que se decidio colocar pernos con un tope para que sirvan como soportes.

\section{Simulación en Software de Elemento Finito.}

Para poder realizar la simulación con el software de elemento finito se debe de cargar el modelo en 3D hecho anteriormente, en la Figura 3 se muestra el modelo 3D cargado en el softwarede elemento finito, así también se deben agregar los valores correspondientes para el material de aluminio 6061 .

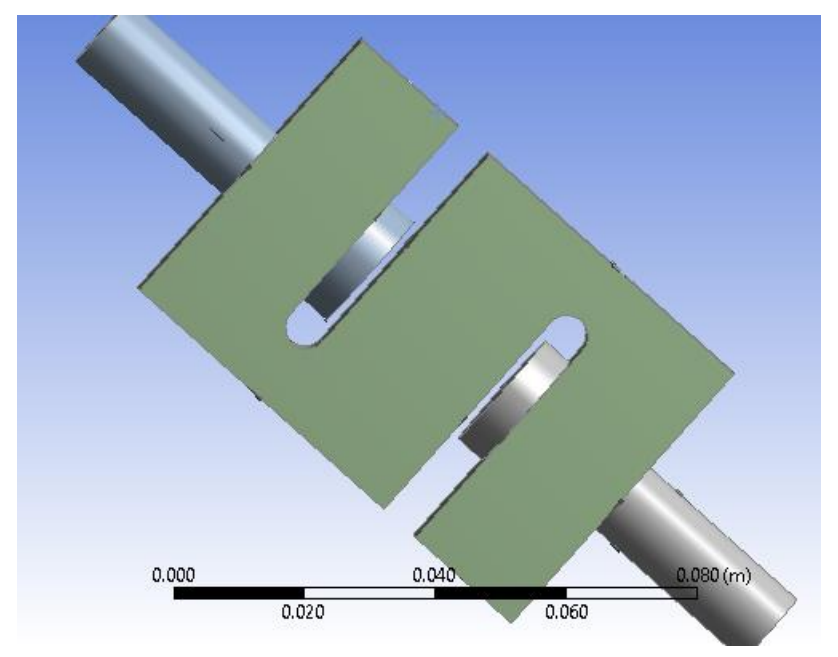

Figura 3 Modelo 3D cargado en el software de elemento finito

Una vez que el modelo 3D esta cargado y los datos del material han sido ingresados, se pasa a colocar las restricciones y las cargas sobre el modelo sólido, esto se muestra en la Figura 4.

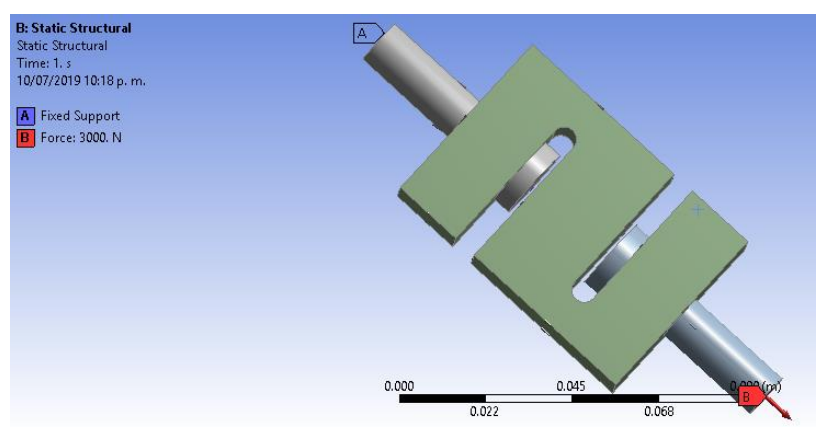

Figura 4 Aplicación de soportes y cargas en el modelo 3D

En el perno superior se coloco un soporte axial para evitar el desplazamiento, mientras que en el perno inferior se aplicó una fuerza de 294 $\mathrm{N}$.

Para el mallado del modelo 3D es necesario hacer un análisis de convergencia, este empezará en un tamaño de elemento de la malla de $0.008 \mathrm{~m}$ y por limitaciones de la máquina con que se esta trabajando el tamaño de elemento final fue de $0.0006 \mathrm{~m}$, en el gráfico 1 se muestran los datos obtenidos entre el tamaño de elemento y el esfuerzo principal.

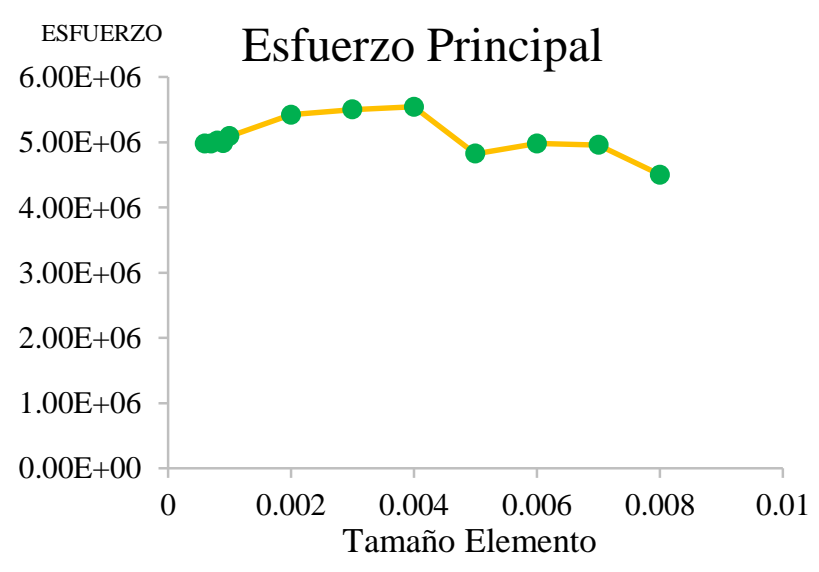

Gráfico 1 Gráfica correspondiente al tamaño de elemento vs esfuerzo principal

Con los datos de la convergencia se puede apreciar que ya no existe mucha diferencia entre los últimos valores, quedando la malla como se muestra en la Figura 5, Figura 7 y Figura 8.

En la Figura 5 se muestra la malla resultante sobre el modelo en $3 \mathrm{D}$, mientras que en la Figura 6 y Figura 7 se muestran acercamientos a la malla. 


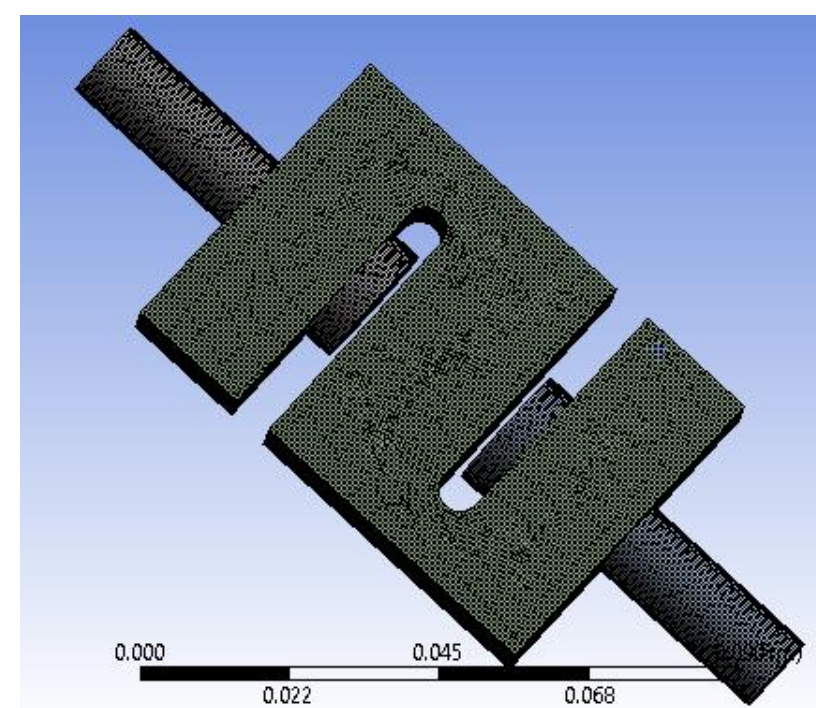

Figura 5 Modelo con la malla aplicada.

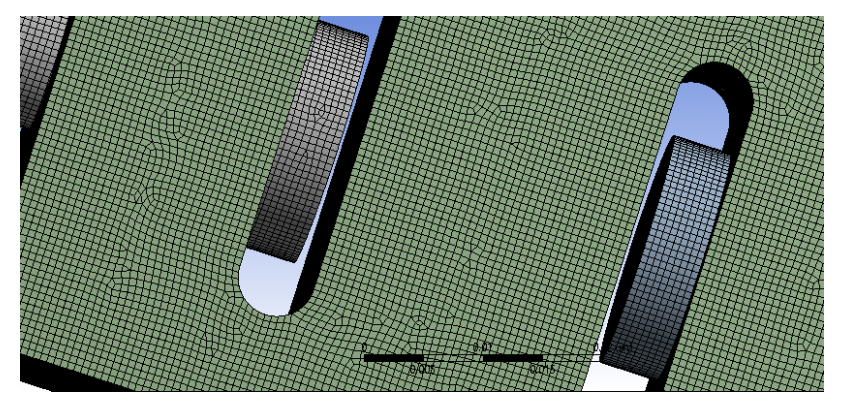

Figura 6 Acercamiento a la malla

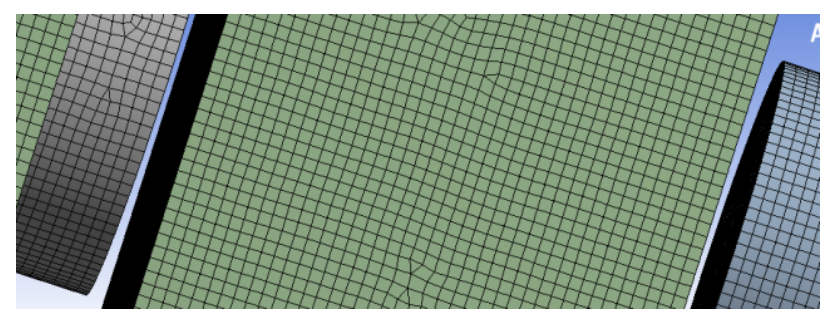

Figura 7 Acercamiento mayor a la malla

Los datos que se quieren obtener con la simulación son: la deformación total que se muestra en la Figura 8, deformación en el eje X mostrada en la Figura 9, deformación en el eje Y representada en la Figura 10, el esfuerzo equivalente de von-mises visto en la Figura 11 y el esfuerzo principal observado en la Figura 12.

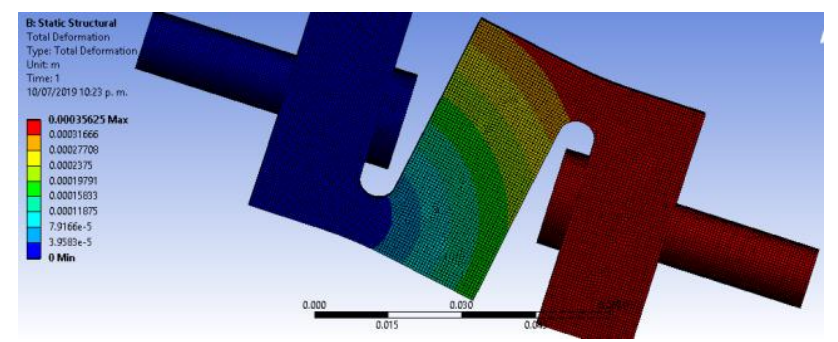

Figura 8 En esta imágen se aprecia la deformación total de la celda, siendo el valor mínimo de $0 \mathrm{~m}$ y máximo de $0.00035625 \mathrm{~m}$

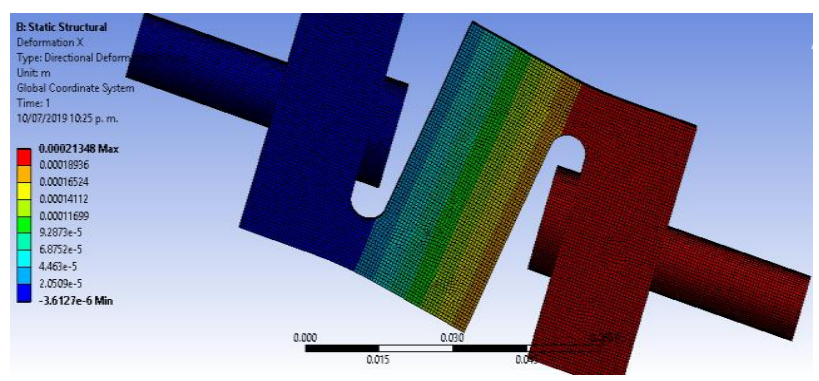

Figura 9 En esta imágen se aprecia la deformación en $X$ de la celda, siendo el valor mínimo de $-3.6127 \times 10^{\wedge}-6 \mathrm{~m}$ y máximo de $0.00021348 \mathrm{~m}$.

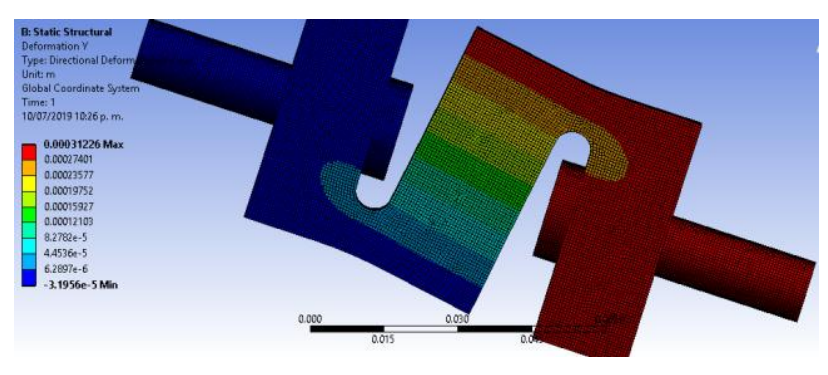

Figura 10 En esta imágen se aprecia la deformación en $\mathrm{Y}$ de la celda, siendo el valor mínimo de $-3.1956 \times 10^{\wedge}-5 \mathrm{~m}$ y máximo de $0.00031226 \mathrm{~m}$.

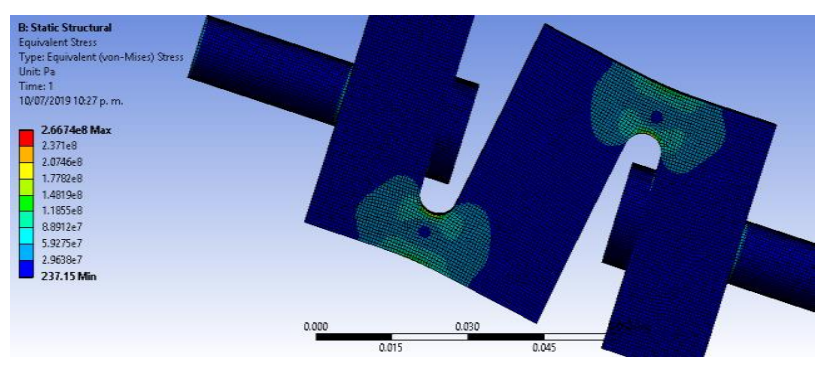

Figura 11 En esta imágen se aprecia el esfuerzo equivalente de Von-Mises en la celda, siendo el valor mínimo de 237.15 pa y máximo de $2.6674 \times 10^{\wedge} 8$ pa.

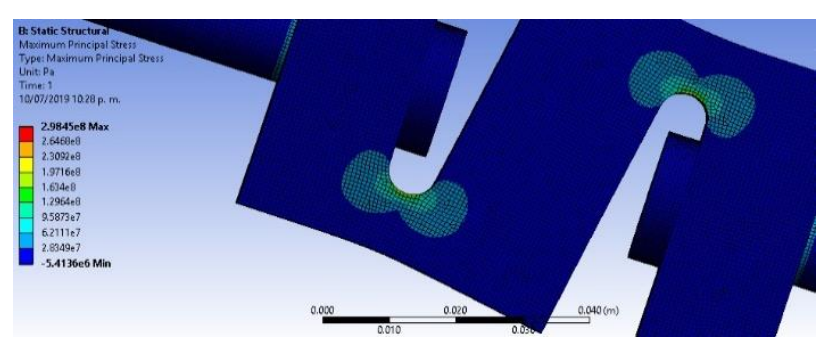

Figura 12 En esta imágen se aprecia el esfuerzo principal en la celda, siendo el valor mínimo de $-5.4136 \times 10^{\wedge} 6$ pa y máximo de $2.9845 \times 10^{\wedge} 8$ pa.

\section{Modelo Físico}

Ya teniendo el modelo en 3D con los resultados pertinentes, ahora es necesario comparar los resustados obtenidos contra un modelo físico. Para esto se tuvo que manufacturar el perfil de una celda de carga tipo "S" con las especificaciones antes mencionadas.

ROMERO-FRANCO, Martín, LÓPEZ-GARZA, Víctor, MARÍNTÉLLEZ, Paulina y PÉREZ-JUÁREZ, Erik. Manufactura y pruebas de una celda de carga para un banco de pruebas de aspas de turbinas eólicas de baja capacidad. Revista de Ingeniería Mecánica. 2019. 
Como se muestra en la Figura 13, para posteriormente hacer uso de galgas extensiométricas, vista en la Figura 14, que se colocaran en su superficie como en la Figura 15 y Figura 16, posteriormente se le agregaran cargas obteniendo y recolectando las mediciones necesarias.

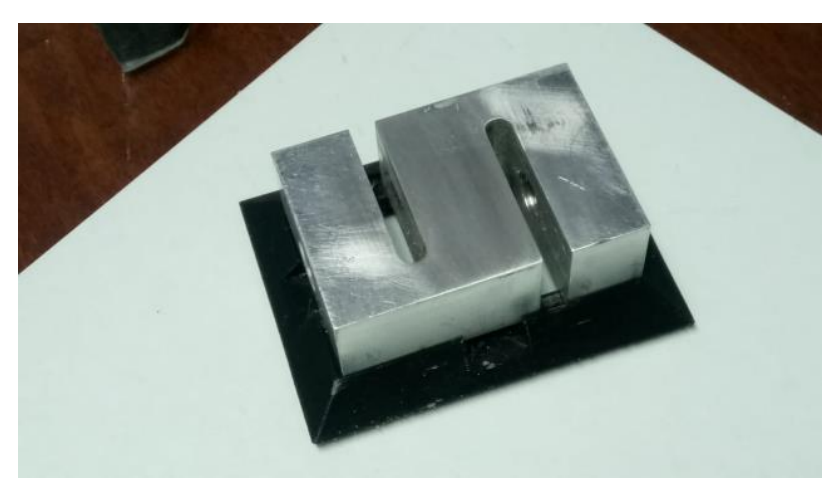

Figura 13 Celda de carga manufacturada.

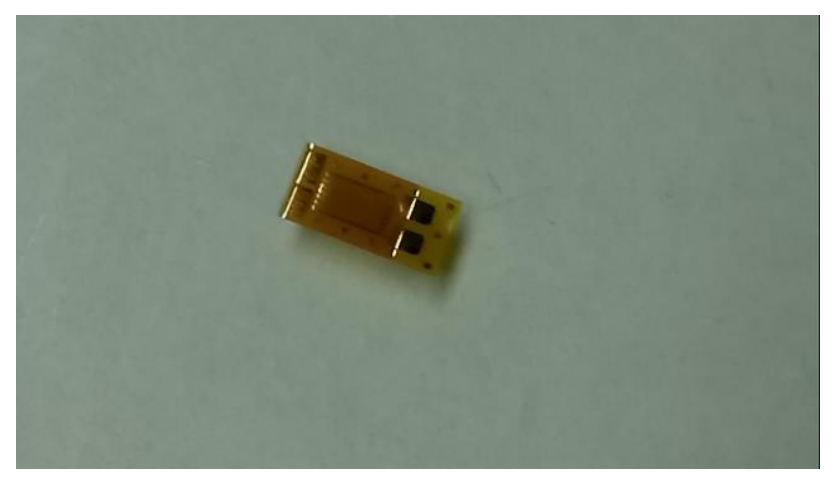

Figura 14 Galga extensiométrica modelo EA-06-120LZ120/E.

A continuación, se lista el material necesario para la colocación de las galgas extensiométricas:

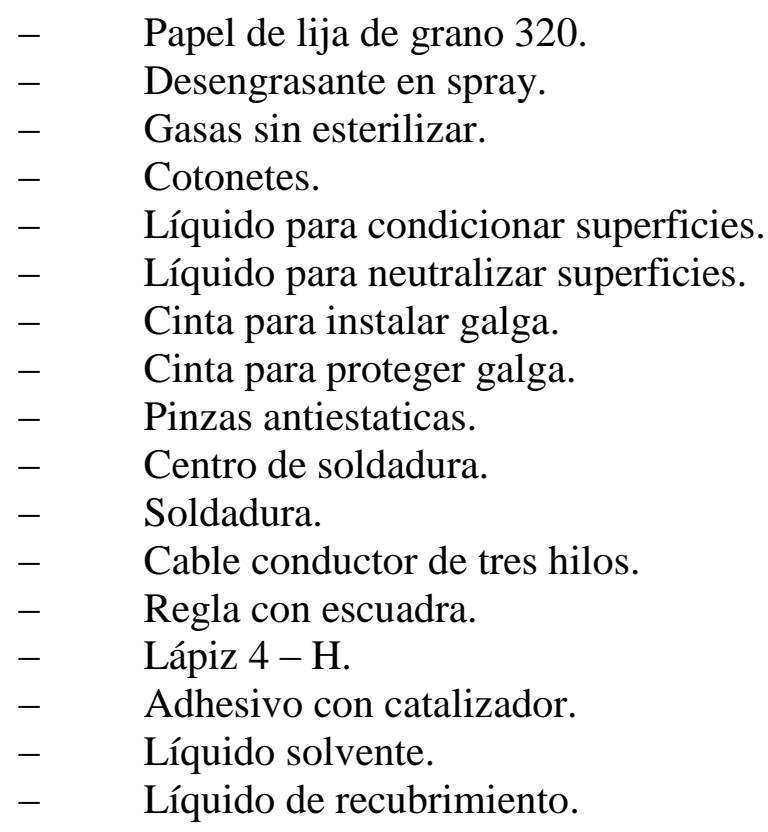

Para la colocación de las galgas extensiométricas se seguirán los siguientes pasos:

\section{Preparación de la superficie}

Primero se trabajará sobre una superficie de vidrio, la cual se le verterá un poco del liquido neutralizante y secara con una gasa limpia (las gasas solo se usarán una vez y se desecharan). Después se utilizará el desengrasante sobre la superficie de la zona de la celda de carga donde se colocará la galga y limpiará con una gasa limpia.

Ahora se lijará la superficie de la celda de carga utilizando la lija de grano 320 y el líquido condicionador como lubricante, limpiando al final con una gasa limpia.

Lo siguiente es trazar una línea guía usando el lápiz 4-H guiandose con la regla, posteriormente limpiar la línea trazada con un cotonete empapado con el liquido condicionador y secar con una gasa limpia. Para iniciar se limpiará la superficie con el líquido neutralizador y un cotonete para después secar con una gasa limpia.

\section{Colocación de la galga extensiométrica}

Limpiar nuevamente la superficie de trabajo con líquido neutralizante y secar con una gasa limpia, usanso esa misma gasa para limpiar las pinzas antiestáticas.

Con cuidado y usando las pinzas antiestáticas se retira el folder plástico donde se encuentra la galga extensiométrica en el empaque, luego de saca del folder plástico con mucho cuidado y se coloca en la superficie del vidrio previamente neutralizada con las terminales viendo hacia arriba.

Con ayuda de la cinta para instalar la galga está se recogerá del vidrio y transportará a la superficie de la celda de carga, donde se colocará guiándose por la marca hecha previamente con el lápiz 4-H.

Haciendo un dobladillo la cinta, se dará media vuelta a la galga extensiométrica, quedando las terminales viendo hacia abajo, se le aplicará una ligera capa de catalizador a la superficie expuesta de la galga y se dejará pasar un minuto. 
Después del minuto se colocará una gota de pegamento en el área donde quedará la galga y se deshará el dobladillo a la cinta para volver a posicionar la galga, con ayuda de una gasa limpia se dará una pasada de abajo hacia arriba de la cinta para eliminar posibles burbujas de aire.

Inmediatamente se debe colocar el pulgar sobre la galga y esperar un minuto, para poder retirar el pulgar, se deberá girar mientras se levanta y esperar dos minutos.

Después de los dos minutos se podrá retirar la cinta de la galga extensiométrica.

\section{Soldado de cables a la galga extensiométrica.}

Primero se cortará un tramo de cable de tres hilos de aproximadamente $40 \mathrm{~cm}$ de largo.

Luego se deben pelar los extremos del cable aproxidamente a $3 \mathrm{~cm}$ de la punta, en un extremo se separarán los hilos negro, blanco y rojo, en el otro extremo se juntarán los hilos negro y blanco, rel rojo permanecerá solo, ya separados se deben torcer los cables.

Ya separados y torcidos los hilos del cable, se les aplicará soldadura, esto se logra pasando los cables por la punta del cautín del centro de soldadura y agregando soldaura como se vaya necesitando.

Lo siguiente es colocar soldadura en las terminales de la galga extensiométrica, para esto se coloca un poco de soldadura en la punta del cautín, se presiona contra la terminal de la galga agregando un poco más soldadura, se debe de tener cuidado de no crear un puente entre las terminales.

El extremo del cable donde se juntaron los hilos negro y blanco, es el que se soldará a la galga extensiométrica, para esto se presentará el extremo del cable a las terminales de la galga, luego se abrirá o cerrará la separación del extremo del cable de ser necesario.

Se cortarán las puntas del extremo del cable casi al raz.

Se tomará un pedazo de cinta para proteger la galga; y se pegará en el extremo del cable, dejando suficiente espacio antes de la punta a soldar.
Tomado el cable por la cinta, se acomodará de tal forma que el extremo del cable a soldar quedé haciendo contacto con las terminales de la galga.

Ya colocado se pasa a soldar presionando el cautín contra el cable y el terminal agregando soldadura, repetir lo mismo con el otro terminal.

Lo siguiente es limpiar la galga y retirar la cinta utilizando solo la brocha del líquido solvente.

Ahora con cuidado se hará un arco al cable y colocará cinta después del arco, esto es para evitar el daño de la galga por algún tirón del cable.

Para terminar, se aplicará liquido de recubrimiento a la galga extensiométrica y en la superficie alrededor de esta, cuando se seque se generará una capa que protegerá la galga.

A continuación, en la Figura 15 y Figura 16 se muestra la posición de como quedaron colocadas las galgas extensiométricas para medir deformación en el eje X y el eje Y.

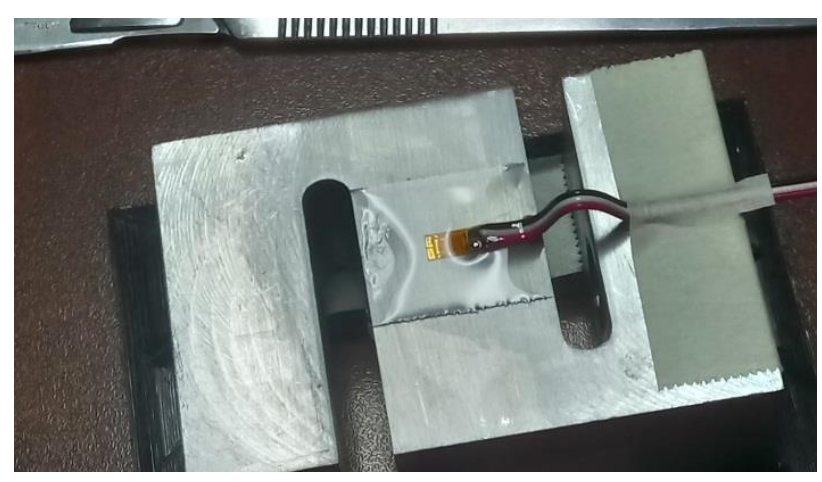

Figura 15 Galga posicionada para optener lecturas en el eje $X$

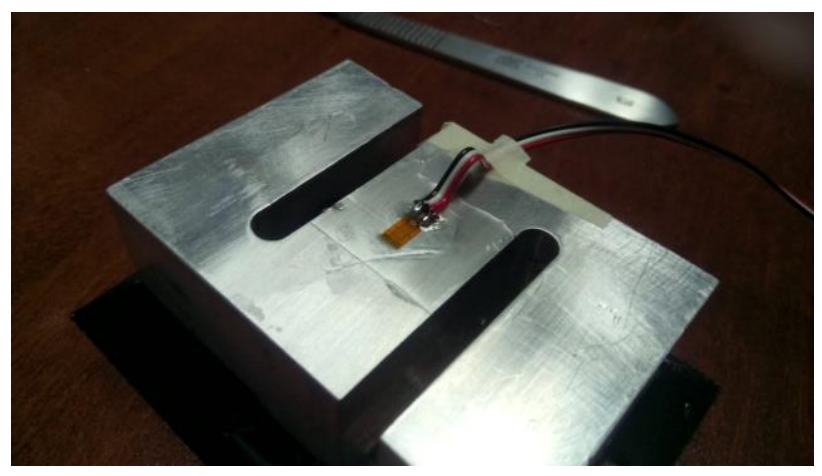

Figura 16 Galga posicionada para obtener lecturas en el eje $\mathrm{Y}$

ROMERO-FRANCO, Martín, LÓPEZ-GARZA, Víctor, MARÍNTÉLLEZ, Paulina y PÉREZ-JUÁREZ, Erik. Manufactura y pruebas de una celda de carga para un banco de pruebas de aspas de turbinas eólicas de baja capacidad. Revista de Ingeniería Mecánica. 2019. 


\section{Conexión con el equipo de medición P3}

En la Figura 17, Figura 18 y Figura 19 se muestra el equipo de medición P3, el cual se conectará a las galgas extensiométricas para obtener los datos de la deformación.

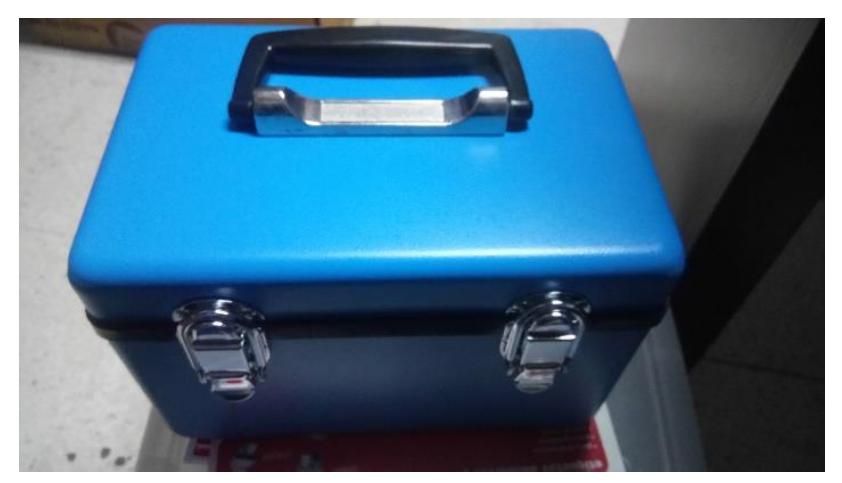

Figura 17 Equipo de medición P3 cerrado

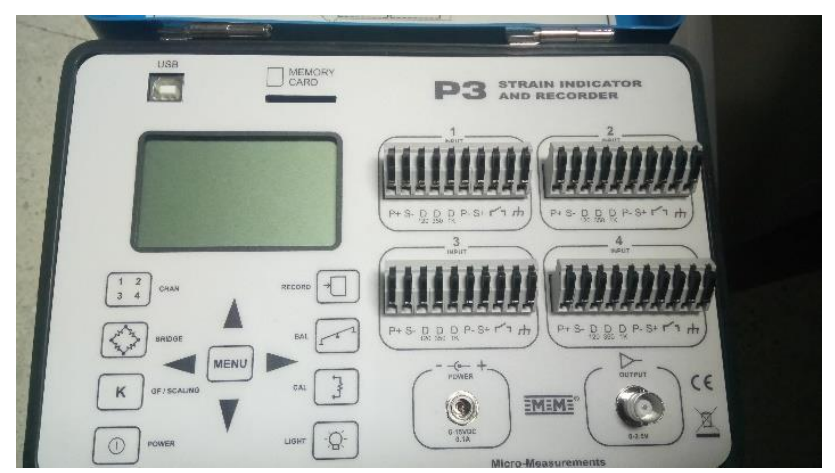

Figura 18 Tablero de control del equipo de medición P3

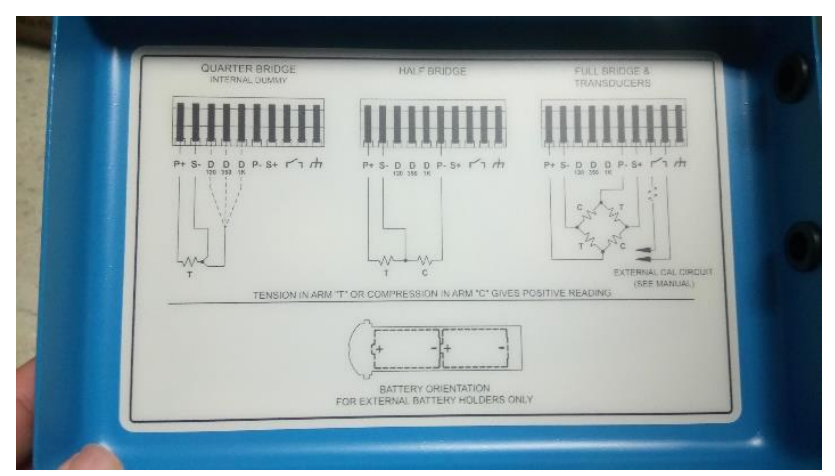

Figura 19 Diagrama de puente de Wheatstone impreso al reverso de la tapa del equipo de medición P3

Antes de hacer las conexiones se debe tener en cuenta que el puente de Wheatstone se compone de cuatro resistencias, como se usarán dos resistencias (galgas) una para medir la deformación en el eje $\mathrm{X}$ y otra para la deformación en el eje $\mathrm{Y}$, se programará el equipo de medición para medio puente.

Se usarán las entradas 1 y 2 del equipo de medición P3 para conectar las galgas extensiométricas, la entrada 1 para medir el eje $\mathrm{X}$ y la entrada 2 para el eje $\mathrm{Y}$.
El orden de los cables será rojo, negro y blanco ordenados de izquierda a derecha para conectarlos en ese mismo orden de las entradas del equipo de medición P3 desde el extremo izquierdo, las conexiones del equipo P3 se muestran en la Figura 20.

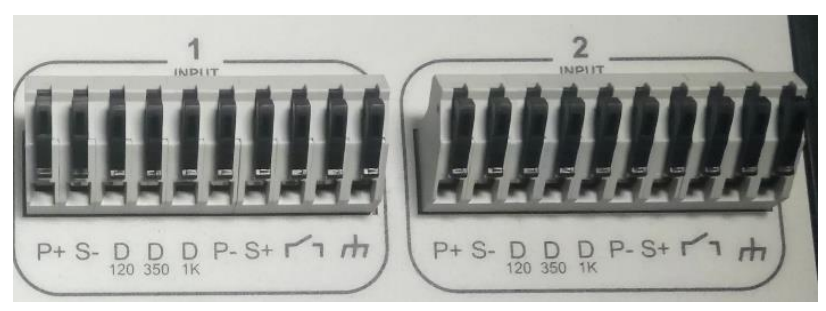

Figura 20 Clemas de conexión de las entradas 1 y 2 del equipo de medición P3

Ya habiendo conectado las galgas al equipo de medición P3, se oprimirá el botón de "POWER" para encender el equipo.

Después se oprimirá el botón de "CHAN" y se revisa que los canales 1 y 2 esten activados y los canales 3 y 4 esten desactivados, si no es así corregir los canales correspondientes.

Para empezar a obtener datos se oprimirá el botón "BAL" balanceando así las galgas extensiométricas dejándolas con valor de 0 .

Se agrega el peso correspondiente, se espera a obtener la medición y se registra.

Se debe de repetir la operación "BAL" para realizar cada medición.

Las mediciones se llevaron a cabo en un taller de maquinado, esto por el espacio y el material que se puede usar como peso que se encuentra en dicho taller. En la Figura 21 se puede ver el equipo de medición P3 conectado a las galgas mientras se realiza la medición,

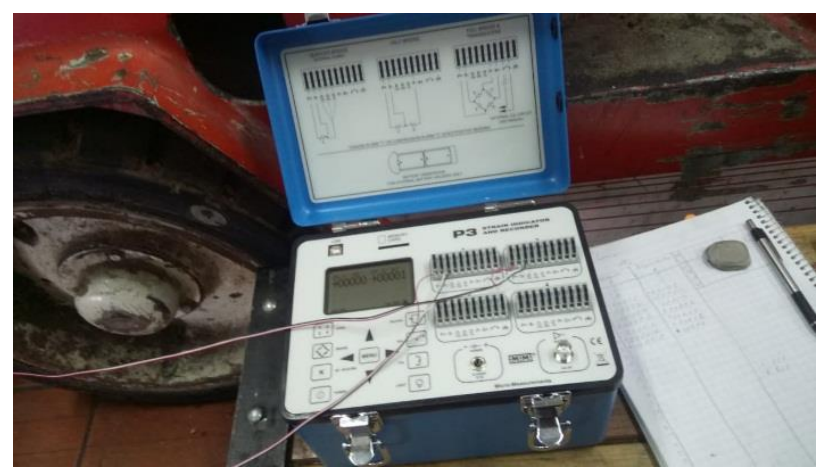

Figura 21 Equipo de medición P3 conectado a las galgas extensiométricas

ROMERO-FRANCO, Martín, LÓPEZ-GARZA, Víctor, MARÍNTÉLLEZ, Paulina y PÉREZ-JUÁREZ, Erik. Manufactura y pruebas de una celda de carga para un banco de pruebas de aspas de turbinas eólicas de baja capacidad. Revista de Ingeniería Mecánica. 2019. 
En la Figura 22 se aprecia el arreglo final de la celda de carga con todos los pesos finales sumando $30 \mathrm{~kg}$ en total.

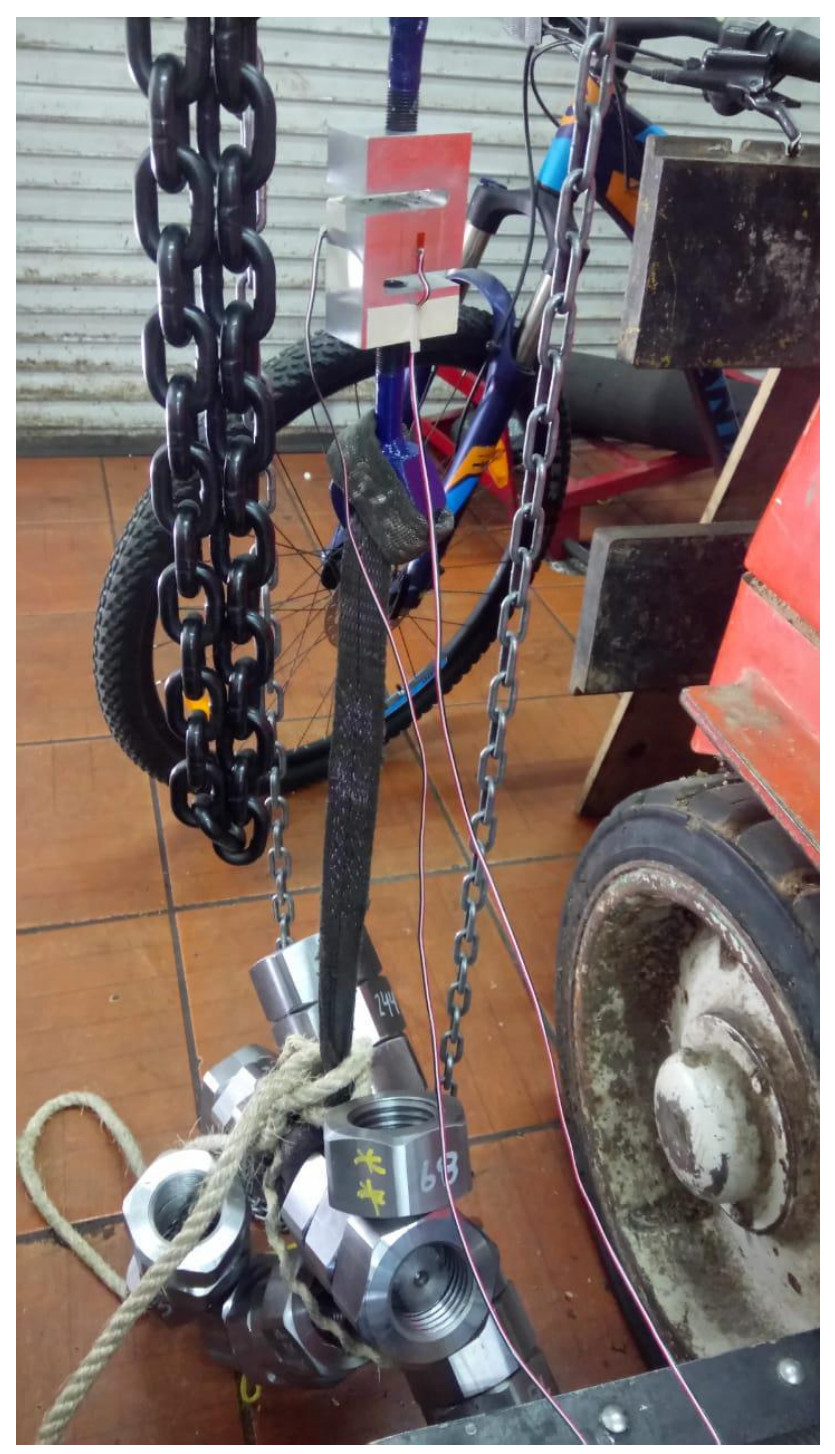

Figura 22 Arreglo de pesos añadido a la celda de carga para realizar la medición

A continuación, se muestra la tabla 1, la cual contiene los datos obtenidos en relación de peso-deformación de las pruebas que se realizarón utilizando la celda de carga.

Dichoas datos están representados gráficamente en la gráfica 2, donde se puede apreciar la tendencia de la deformación respecto con el aumento del peso sobre la celda de carga.

\begin{tabular}{|c|c|c|}
\hline $\begin{array}{c}\text { Peso } \\
\mathrm{Kg}\end{array}$ & Deformacion X & Deformacion Y \\
\hline 0 & -0.00000 & -0.00000 \\
\hline 1 & -0.00000 & -0.00000 \\
\hline 2 & -0.00001 & -0.00001 \\
\hline 3 & -0.00002 & -0.00002 \\
\hline 4 & -0.00004 & -0.00004 \\
\hline 5 & +0.00001 & +0.00000 \\
\hline 6 & +0.00001 & +0.00000 \\
\hline 7 & +0.00001 & +0.00000 \\
\hline 8 & +0.00000 & +0.00001 \\
\hline 9 & +0.00000 & +0.00001 \\
\hline 10 & +0.00000 & +0.00001 \\
\hline 11 & +0.00000 & +0.00002 \\
\hline 12 & +0.00000 & +0.00002 \\
\hline 13 & +0.00001 & +0.00003 \\
\hline 14 & +0.00001 & +0.00003 \\
\hline 15 & +0.00001 & +0.00003 \\
\hline 16 & +0.00001 & +0.00003 \\
\hline 17 & +0.00001 & +0.00003 \\
\hline 18 & +0.00001 & +0.00004 \\
\hline 19 & +0.00001 & +0.00004 \\
\hline 20 & +0.00002 & +0.00005 \\
\hline 21 & +0.00002 & +0.00005 \\
\hline 22 & +0.00003 & +0.00006 \\
\hline 23 & +0.00004 & +0.00006 \\
\hline 24 & +0.00004 & +0.00006 \\
\hline 25 & +0.00004 & +0.00007 \\
\hline 26 & +0.00004 & +0.00007 \\
\hline 27 & +.0 .00004 & +0.00008 \\
\hline 28 & +0.00005 & $\begin{array}{r}+0.00009 \\
\end{array}$ \\
\hline 29 & +0.00005 & +0.00009 \\
\hline 30 & +0.00006 & +0.00009 \\
\hline
\end{tabular}

Tabla 1 Datos obtenidos por la medición en la celda de carga

\section{Deformación en X y Y}

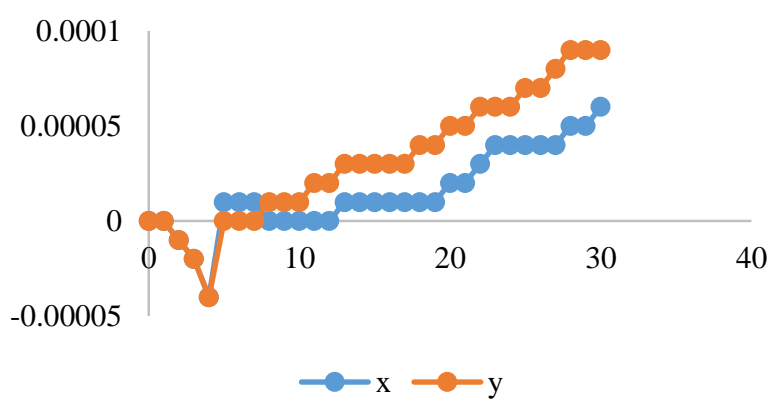

Gráfica 2 Tendencia de la deformación en el eje X y Y.

\section{Resultados}

Ya teniendo los valores tanto de la simulación del modelo matemático, así como las mediciones obtenidas del modelo físico, ahora es posible la comparación entre ambos.

Para realizar esta comparación solo se tomarán en cuenta los valores finales, los cuales son:

ROMERO-FRANCO, Martín, LÓPEZ-GARZA, Víctor, MARÍNTÉLLEZ, Paulina y PÉREZ-JUÁREZ, Erik. Manufactura y pruebas de una celda de carga para un banco de pruebas de aspas de turbinas eólicas de baja capacidad. Revista de Ingeniería Mecánica. 2019. 


\begin{tabular}{|l|c|c|}
\hline \multicolumn{1}{|c|}{ Modelo } & Def. X & Def. Y \\
\hline Matemáti-Co & 0.00021348 & 0.00031226 \\
\hline Físico & 0.00006 & 0.00009 \\
\hline Diferencia & 0.00015348 & 0.00022226 \\
\hline
\end{tabular}

Tabla 2 Valores finales de la simulación y medición de la celda de carga

\section{Conclusiones}

Con los datos obtenidos de la simulación y las mediciones tomadas mediante el equipo de medición P3 y las galgas extensiométricas colocadas en una celda de carga de aluminio 6061, se puede observar que existe una diferencia notable en los resultados ya que la simulación del modelo 3D muestra una mayor deformación que el modelo físico, también se debe mencionar que en el modelo físico en las primeras mediciones se registraron valores negativos de deformación, es hasta después de los $5 \mathrm{~kg}$ que las mediciones se desplazan en sentido positivo, pero pasa de -0.00004 a +0.00000 , lo cual puede indicar que se debe a una contracción del material por temperatura, alguna contaminación en la superficie de la galga extensiométrica o que no este bien pegada la galga.

\section{Agradecimientos}

Universidad Michoacana de San Nicolás de Hidalgo.

Facultad de Ingeniería Mecánica.

Taller de maquinado y mantenimiento industrial SILVER.

\section{Referencias}

[20] Monje, C. (2011). Metodología de la investigación cuantitativa y cualitativa, guía didáctica, Neiva, Colombia: Universidad Surcolombiana.

AMDEE (Asociación Mexicana de Energía Eólica) (2018), "El viento en números", https://www.amdee.org/el-viento-en-numeros, 20 de febrero del 2019.

Anup KC, Jonathan Whale y Tania Urmee (2019). Urban wind conditions and small wind turbines in the built environment: A review,131, 268-283.
Clemence Rubiella, Cyrus A. Hessabi y Arash SoleimanFallah (2018). State of the art in fatigue modelling of composite wind turbine blades. Elsevier,117, 230-245.

D.J. Willis, C. Niezrecki, D. Kuchma, E. Hines y S.R. Arwade (2018). Wind energy research: State-of-the-art and future research directions. Elsevier,125, 133-154.

David Wood, Small Wind turbines, Analysis, Design, and Application. Springer-Verlag London Limited 2011.

Erich Hau, Fundamentals, Technologies, Application, Economics, Second Edition, Springer-Verlag Berlin Heidelberg 2006.

Erick Pérez Juárez (2019). Construcción de una máquina de ensayo a fatiga para álabes de aerogeneradores de baja capacidad (tesis de maestría). Universidad Michoacana de San Nicolás de Hidalgo.

Erick Yuvaniel Gómez Urieta (2012). Diseño y manufactura del Hub y álabes de una turbina de viento (tesis de maestria). Universidad Michoacana de San Nicolas de Hidalgo.

Ferrero, Jóse María. Guijarro, E. Instrumentación Electrónica. Sensores. España, servicio de publicaciones UPV, 1994.

Germanischer Lloyd, Hamburg, Germany: "the new guideline for the certification of wind turbines", Edition 2010.

H. J. Sutherland, On The Fatigue Analysis of Wind Turbines. United States, 1999.

Hak GuLee y JungwanLee (2018). Measurement theory of test bending moments for resonancetype fatigue testing of a full-scale wind turbine blade. Elsevier,200, 306-312.

Hong-Kwan Kim, Jang-Ho Lee, Se-Myong Jang y Ki-Weon Kang (2013). Structural Analysis and Testing of $1.5 \mathrm{~kW}$ Class Wind Turbine Blade. Journal of Fluid Machinery,13, 37-44.

IEC 61400-2 "Wind Turbines - Part2: Design requirements of small wind turbines", Edition 2015. 
IRENA (2017), REthinking Energy 2017: Accelerating the global energy transformation. International Renewable Energy Agency, Abu Dhabi.

J.H. Wu y F.M. Lai (2014) Fatigue Life Analysis of Small Composite Sandwich Wind Tuebine Blades, 14, 2014-2020.

Jean-Daniel Pitteloud and Stefan Gsänger. World Wind Energy Association. (2017). Small Wind World Report small wind reportw. WWEA, Bonn, Germany.

L.Menegozzo, A. Dal Monte, E. Benini y A. Benato (2018) Fatigue Small wind turbines: A numerical study for aerodynamic performance assessment under gust conditions, 121, 123-132. Leyva Huitrón, R. (2019). Investigación de materiales para celdas solares sensibilizadas por colorante.

Mejía López, J. A., Ruiz Guzmán O. A., Gaviria Ocampo, I. N., \& Ruiz Guzmán, C. P. (2019). Aplicación de metología "DESING THINKING" en el desarrollo de cortadora automática CNC para MIPYMES de confección. Revista UIS Ingenierias, 18(3), 157-168.

Phellan, S., \& Kevin, W. (2019). Ingeniería inversa y diseño de la automatización de un dosificador de polvos, utilizando celda de carga a través de microcontrolador atmega para la empresa Jacs.

Quintero Marín, M. (2019). Análisis pesadoras dinámicas.

REN21, 2018, Renewables 2018 Global Status Report, (Paris: REN21 Secretariat) ISBN 978-39818911-3-3.

Rosel-Solís, M. J., Molina-Salazar, J., DávalosRamírez, J. O., Pimentel-Mendoza, A. B., \& Vega, Y. (2019). Análisis de características de materiales compuestos construidos sobre núcleos de ABS preparados por fabricación aditiva. DYNA-Ingeniería e Industria, 94(3).

Sina Yasin Erturk (2014). Tower design load verification of $3.5 \mathrm{~kW}$ wind turbine (tesis de maestria). University of Wyoming.

Tony Burton, David Sharpe, Nick Jenkins, Ervin Boss Anyi (2001). Wind Energy Handbook, John Wiley \& Sons, Ltd.
Yuan-KangWua, Huei-JengLinb y JhihHaoLinb (2019). Certification and testing technology for small vertical axis wind turbine in Taiwan. Elsevier,31, 34-42.

Z. Pan and J. Wu, A Novel Multi-Point Excitation Fatigue Testing Method for Wind Turbine Rotor Blades, vol. 10. 2017. 